\title{
The Burden of Unavailable Drugs Cost to Patients Admitted to Internal Medicine Ward of Tikur Anbessa Specialized Hospital
}

\section{Misgana Ayele Hussien \\ Eyerusalem Berhanemeskel \\ Haile \\ Bezawit Negash Demisse (D)}

Department of Pharmaceutics and Social Pharmacy, School of Pharmacy, College of Health Sciences, Addis Ababa University, Addis Ababa, Ethiopia
Correspondence: Bezawit Negash Demisse

Email bezawit.negash@aau.edu.et
Purpose: To assess the burden of unavailable drugs cost to patients admitted to TikurAnbessa Specialized Hospital's internal medicine ward. Costs of medications obtained inside and outside the pharmacy and other burdens associated with drugs' unavailability are also estimated and explored.

Patient and Methods: A facility-based cross-sectional study design was conducted from November 9 to November 23, 2020. Quantitative data on medication costs were collected from patients admitted to TikurAnbessa Specialized Hospital internal medicine ward B8. Qualitative information was collected from patients, caregivers, and health care professionals through interviews to assess the burdens of unavailability of pharmaceuticals on patients and the health care system.

Results: A total of 32 patients were enrolled in the study. Participants' age ranged from 14 to 92. Eighteen (56.25\%) paid in cash for medications bought within the hospital pharmacy, while the remaining $14(43.75 \%)$ were treated for free. A total of 68 medicines were prescribed during the study period. Of this, $33(48.53 \%)$ medicines were not available in the hospital pharmacy. The total costs of patients were $78.07 \$$ and $1530.84 \$$ when purchased from inside and outside the hospital pharmacy, respectively. The average price of medications per patient ranged from 0.025 to $8.41 \$$ inside the hospital pharmacy, where the average cost per patient outside the hospital pharmacy ran from 0.128 to $54.67 \$$. For the qualitative part, four patients and/or caregivers and five health care professionals were interviewed, and three key themes emerged from the thematic analysis: (i) awareness of the availability of medication, (ii) management of the burden of stock out, and (iii) impact of the stock out.

Conclusion: The study came up with a result that the hospital pharmacy has high unavailability of medication. Patients complain about unwanted and unreasonably expenditure to purchase pharmaceuticals from outside pharmacies and associated delayed treatment.

Keywords: burden, unavailability, hospital pharmacy

\section{Introduction}

Pharmacy service is an essential component of health care delivery in hospitals. It contributes to improved treatment outcomes by ensuring availability and rational use of quality, safe and effective medicines. Provision of effective pharmacy service promotes optimal use of scarce resources, thereby improving quality of care, resulting in better health outcomes. The eventual success of hospital pharmacy service is to achieve overall patient satisfaction by enhancing medicines, premises, and workflow and providing quality pharmaceutical services. ${ }^{1}$ 
In low and middle-income countries, patients may go to more expensive private health care settings due to inaccessible public health care facilities, inadequate availability of medicines and perceived image of public health care settings as low-quality diagnosis and treatment. ${ }^{2}$ Like other developing countries, in Ethiopia, public health care is provided at nominally low prices and free to those that cannot afford to pay. But the health care consumer population still has to make a considerable amount of out-ofpocket health expenditure, especially to purchase from the private sector due to the unavailability of medicines in the public health care settings. ${ }^{3}$

Governments in low- and middle-income countries (LMICs) are increasingly advocating for universal health coverage. One of the critical components of any functional health system is the availability of medicines. However, the majority of the population in LMICs have limited access to medicines. Even when medicines are available, patients have to pay for them out-of-pocket, the cost contributing up to $60 \%$ of their health care expenditure. ${ }^{4}$

From anecdotal observation, when pharmaceuticals are not available in TikurAnbessa Specialized Hospital wards, patients are forced to buy medicines from private retail pharmacies. This medication unavailability poses a financial burden and other health-related problems to patients, their caregivers, and health care professionals.

Therefore, this study aimed to show the financial burden that patients and caregivers admitted to TikurAnbessa Specialized Hospital's (TASH) internal medicine ward due to pharmaceuticals' unavailability within the hospital pharmacy. Moreover, it also shows other health-related burdens that patients, caregivers, and healthcare professionals face.

\section{Materials and Methods Study Setting}

The study was conducted at TASH. TASH was inaugurated in 1972. It is a 700-bed tertiary care teaching hospital of Addis Ababa University. This hospital offers diagnosis and treatment for approximately $370,000-400,000$ patients per year. On the other hand, as the country's largest teaching hospital, it trains a large number of undergraduate and graduate students. It is also an institution where specialized clinical services not available in other public or private institutions are rendered to the whole nation. TASH internal medicine department has 96 beds comprising of 4 wards, namely B8, C8, D8, and B5, which are cardiovascular and neurologic, renal, hematology, and gastrointestinal wards, respectively.

\section{Study Design and Period}

A facility-based cross-sectional study design supplemented by quantitative and qualitative approaches was employed for two weeks, from November 8 to November 21, 2020.

\section{Sampling and Sample Size Determination}

The study used a convenient sampling method to select among the four internal medicine wards of TASH. Since Ward B8 has a large number of beds and is composed of different groups of respondents that can provide relevant information about the burdens of pharmaceuticals' unavailability, it was chosen for this study to select patients, caregivers and health care professionals as key informants for the qualitative data.

For quantitative data, all patients admitted to B8 Ward, and their caregivers were considered sample populations.

\section{Data Collection Procedure}

Relevant information on patients' socio-demographic characteristics was obtained using a semi-structured questionnaire. A data abstraction tool was used to collect secondary data from patient charts such as clinical characteristics (disease condition, duration of hospital stay), number, and type of medications. The purchased medications and their cost were collected daily by physically asking the patient or the caregiver. Two separate interview guides for patients/caregivers and the health care professionals were used to collect the qualitative data (Please refer the interview guide questions in the Supplementary File). The interview guides were carefully translated to Amharic and back-translated to English to assure consistency.Interviews were recorded while relevant points were note taken. Content validity was checked for the data collection tool by academicians.

\section{Data Analysis}

After checking the data's completeness and consistency, Microsoft Excel 2016 was used to analyze the descriptive statistics. The descriptive result is presented by using tables. The qualitative data were transcribed and translated verbatim using Microsoft (MS) and analyzed using a thematic approach. 


\section{Results}

A total of 32 patients admitted to the B8 ward from November 8 to 21, 2020, enrolled in the study. For the qualitative part, four patients and/or caregivers and five health care professionals were interviewed (Table 1).

\section{Clinical Characteristics of Patients}

Patients disease conditions were variable and widespread, with more than 50 disease conditions diagnosed. Heart failure $(9,28.13 \%)$, hypertension $(7,21.3 \%)$, type II DM (7, 21.3\%), pneumonia $(6,18.75 \%)$, and stroke (6, $18.75 \%$ ) were the common disease conditions.
Duration of hospitalization lasted from 2 to 14 days, with an average of 8 days. Two patients died, whereas one patient was transferred to a different ward. Six patients were hospitalized for 14 days.

Among the patients, $18(56.25 \%)$ paid in cash for their medications. The remaining 14 were served for free, as they are either insured $(11,34.37 \%)$ or eligible for free treatment $(3,9.37 \%)$.

\section{Estimation of Unavailability and Costs of Medications Inside and Outside of TASH} A total of 68 medications were prescribed for the patients. Three-fourth (51) of the prescribed medications were

Table I Socio-Demographic Characteristics of Patients Admitted to Internal Medicine B8 Ward of TikurAnbessa Specialized Hospital, Addis Ababa Ethiopia, November, 2020

\begin{tabular}{|c|c|c|c|}
\hline Socio-Demographics & Category & Frequency & Percentage \\
\hline \multirow[t]{7}{*}{ Age } & $<18$ & 3 & 9.375 \\
\hline & $19-30$ & 6 & 18.75 \\
\hline & $31-40$ & 6 & 18.75 \\
\hline & $4 I-50$ & 7 & 21.87 \\
\hline & $50-60$ & 4 & 12.5 \\
\hline & $61-75$ & 4 & 12.5 \\
\hline & $>75$ & 2 & 6.25 \\
\hline \multirow[t]{2}{*}{ Gender } & Male & 20 & 62.5 \\
\hline & Female & 12 & 37.5 \\
\hline \multirow[t]{3}{*}{ Marital status } & Single & 15 & 46.87 \\
\hline & Married & 14 & 43.75 \\
\hline & Widowed & 3 & 9.375 \\
\hline \multirow[t]{4}{*}{ Level of education } & No formal education & 6 & 18.75 \\
\hline & $\mathrm{I}-8$ & 9 & 28.12 \\
\hline & $9-12$ & 11 & 34.37 \\
\hline & College and above & 6 & 18.75 \\
\hline \multirow[t]{2}{*}{ Residence } & Addis Ababa & 22 & 68.75 \\
\hline & Out of Addis & 10 & 31.25 \\
\hline \multirow[t]{7}{*}{ Occupation } & Government employee & 6 & 18.75 \\
\hline & Private employee & 9 & 28.12 \\
\hline & Own private business & 6 & 18.75 \\
\hline & Retired & 3 & 9.375 \\
\hline & Housewife & 3 & 9.375 \\
\hline & Student & 4 & 12.5 \\
\hline & No job & 1 & 3.125 \\
\hline \multirow[t]{5}{*}{ Monthly Income } & $<25.64 \$$ & 21 & 65.62 \\
\hline & $25.66 \$-51.28 \$$ & 3 & 9.375 \\
\hline & $51.3 \$-102.56 \$$ & 2 & 6.25 \\
\hline & $102.58 \$-128.21 \$$ & 0 & 0 \\
\hline & $>128.23 \$$ & 6 & 18.75 \\
\hline
\end{tabular}

Note: I US $\$=39$ ETB. 
prescribed with a frequency ranging from 1-5, out of which 26 and 15 medications were prescribed once and twice, respectively. The remaining medications were prescribed frequently, ranging from 5-12 times (Table 2).

Of the 68 medications prescribed, 26 (38.23\%) had $100 \%$ availability in the hospital pharmacy. In contrast, $33(48.53 \%)$ of the medicines were not available in the hospital pharmacy, hence considered $100 \%$ stock out in the hospital during the study period.

The remaining nine medications had variable availability; some patients founded the prescribed medications in the hospital pharmacy, whereas others purchased from outside. Amlodipine $10 \mathrm{mg}$ tablet (75\%), Furosemide $40 \mathrm{mg}$ tablet (88.9\%), Metoprolol $50 \mathrm{mg}$ tablet $(20 \%)$, Omeprazole $20 \mathrm{mg}$ injection (50\%), Omeprazole $20 \mathrm{mg}$ capsule (85.71\%), Potassium Chloride $600 \mathrm{mg}$ tablet (85.71\%), Potassium Chloride $2 \mathrm{meq} / \mathrm{mL}$ injection $(50 \%)$ Spironolactone $25 \mathrm{mg}$ tablet $(90 \%)$ and Tramadol $50 \mathrm{mg}$ injection (25\%) availability in the hospital pharmacy.

A patient spent a total of $78.05 \$$ and an average of 4.33 $\$$ to purchase drugs that are available in the hospital pharmacy for eight days of doses. In comparison, they spend a total of $1530.84 \$$ and an average of $54.67 \$$ to purchase drugs from the private market for the same period of doses (Table 3 ).

From the drugs available in the hospital pharmacy, Vancomycin injection was relatively expensive, with a total and average cost of $50.46 \$$ and $8.41 \$$ respectively.
The average costs of medications per patient bought within the hospital pharmacy range from 0.025 to $8.41 \$$ for an average of 8 days of doses (Table 4).

Participants having community insurance and eligible for free treatment did not pay for their medications bought in the hospital pharmacy. Thus, they did not have direct medication costs regarding drugs available in the hospital pharmacy. Since Artesunate tablet, RHZE tablet, and RH tablet are program medications; they are provided to the patients for free. In addition, Digoxin $0.25 \mathrm{mg}$ tablet and Hydrochlorothiazide $25 \mathrm{mg}$ tablet came to the hospital pharmacy through donation. These two medications were provided for free irrespective of the patients' method of payment. The costs of the remaining medications ranged from $0.025-0.717 \$$.

Among the medications bought outside the hospital pharmacy, unfractionated heparin scored the highest total dose cost (367.18\$) for an average of 8 days, while labetalol $5 \mathrm{mg}$ injection was the most expensive with $61.54 \$$ average cost per patient for an average of 8 days of dose followed by Protamine Sulphate $10 \mathrm{mg} / \mathrm{mL}$ injection (56.41\$) and Enoxaparin 60mg injection (52.33\$). The average cost of medication per patient bought outside the hospital pharmacy ranges from 0.128 to $61.54 \$$ for an average of 8 days of dose (Table 5).

The remaining 26 medications costs from $0.128-11.54$ \$. Three medications, Desmopressin 100mcg tablet, Unfractionated heparin 25,000 IU injection and Morphine

Table 2 Frequency of Prescribed Medication in Internal Medicine Ward B8 of Tikur anbessa Specialized Hospital, Addis Ababa, Ethiopia, November 2020

\begin{tabular}{|l|l|l|l|}
\hline No & Medication & Frequency & Percentage \\
\hline 1 & Ceftriaxone Img injection & 12 & 37.5 \\
2 & Spironolactone $25 \mathrm{mg}$ tablet & 10 & 31.25 \\
3 & Furosemide $40 \mathrm{mg}$ tablet & 9 & 28.12 \\
4 & Unfractionated heparin $25,000 \mathrm{IU}$ injection & 9 & 28.12 \\
5 & Vancomycin Img injection & 9 & 28.12 \\
6 & Furosemide 20mg/2mL injection & 8 & 25 \\
7 & Metronidazole $500 \mathrm{mg}$ injection & 8 & 25 \\
8 & Omeprazole 20 mg injection & 8 & 25 \\
9 & $40 \%$ Dextrose injection & 7 & 21.75 \\
10 & Omeprazole 20 mg capsule & 7 & 21.75 \\
11 & Potassium Chloride $600 \mathrm{mg}$ tablet & 7 & 21.75 \\
12 & Digoxin 0.25 mg tablet & 6 & 18.75 \\
13 & Atorvastatin $40 \mathrm{mg}$ tablet & 5 & 15.62 \\
14 & Cefepime Ig injection & 5 & 15.62 \\
15 & Metoprolol $50 \mathrm{mg}$ tablet & 5 & 15.62 \\
16 & Metoclopramide I0mg/2mL injection & 5 & 15.62 \\
17 & Warfarin $5 \mathrm{mg}$ tablet & 5 & 15.62 \\
\hline
\end{tabular}


Table 3 Costs of Medication Bought Inside and Outside the Hospital Pharmacy by Patients Admitted to Internal Medicine Ward of TASH, Addis Ababa, Ethiopia, November 2020

\begin{tabular}{|l|c|c|}
\hline $\begin{array}{l}\text { Cost of } \\
\text { Medication per }\end{array}$ & $\begin{array}{c}\text { Inside the Hospital } \\
\text { Pharmacy (\$) }\end{array}$ & $\begin{array}{c}\text { Outside the } \\
\text { Hospital } \\
\text { Pharmacy (\$) }\end{array}$ \\
\hline Minimum cost & 0.18 & 3.07 \\
Maximum cost & 11.91 & 163.58 \\
Total cost & 78.05 & 1530.84 \\
Average cost & 4.33 & 54.67 \\
\hline
\end{tabular}

Note: I US $\$=39$ ETB.

$10 \mathrm{mg} / \mathrm{mL}$ injection, were stock out outside the hospital pharmacy. As a result of this, Desmopressin 100mcg tablet and Morphine $10 \mathrm{mg} / \mathrm{mL}$ injection were changed to
Hydrochlorothiazide $25 \mathrm{mg}$ tablet and Morphine $30 \mathrm{mg}$ tablet.

\section{Key Themes of the Interviews}

From the analysis, three themes emerged, which are:

1. Awareness of the availability of medication.

2. Management of the burden of stock out.

3. Impact of the stockout.

\section{Awareness of the Availability of Medication}

Patients reported that most medications were not available in the nearby private pharmacies, mainly because of the

Table 4 Costs of Selected Medications Bought Inside the Hospital Pharmacy by Patients Admitted to Internal Medicine B8 Ward of Tikur anbessa Specialized Hospital, Addis Ababa, Ethiopia, November 8-21, 2020

\begin{tabular}{|l|l|c|c|}
\hline No & \multicolumn{1}{|c|}{ Medication } & Total Cost (\$) & Average Cost (\$) per Patient \\
\hline $\mathrm{I}$ & Vancomycin Ig injection & 50.46 & $8.4 \mathrm{I}$ \\
2 & Rivaroxaban I0 mg tablet & 5.05 & 2.52 \\
3 & Furosemide 20mg/2mL injection & 5.61 & 1.87 \\
4 & Tramadol 50 mg injection & 1.54 & 1.54 \\
5 & Ceftriaxone Ig injection & 5.56 & 1.08 \\
6 & Omeprazole 20mg injection & 2 & 1 \\
7 & Potassium Chloride 2meq/mL injection & 0.84 & 0.84 \\
8 & Pyridoxine 50 mg tablet & 1.2 & 0.6 \\
\hline
\end{tabular}

Notes: I US $\$=39$ ETB, The average cost of the medicine per patient is obtained by dividing the sum of the medicine cost from total prescription by the number of prescriptions.

Table 5 Costs of Selected Medications Bought Outside the Hospital Pharmacy by Patients Admitted to Internal Medicine B8 Ward of Tikur Anbessa Specialized Hospital, Addis Ababa, Ethiopia, November 8-21, 2020

\begin{tabular}{|l|l|l|l|}
\hline No & Medication & Total Cost (\$) & Average Cost (\$) per Patient \\
\hline 1 & Labetalol 5 mg injection & 61.54 & 61.54 \\
2 & Protamine sulphate IOmg/mL injection & 56.41 & 56.41 \\
4 & Enoxaparin $60 \mathrm{mg}$ injection & 157 & 52.33 \\
5 & Meropenem Ig injection & 102.18 & 51.09 \\
6 & Cefepime Ig injection & 235.64 & 47.13 \\
7 & Unfractionated heparin 25,000 IU injection & 367.18 & 45.89 \\
8 & Lactulose 333 mg injection & 41.02 & 41.02 \\
9 & Mannitol 20\% infusion & 55.13 & 27.56 \\
10 & Sacubitril/valsartan 49mg/5Img tablet & 18.59 & 18.59 \\
11 & Omeprazole 20mg injection & 40 & 10 \\
12 & Metronidazole 500mg injection & 76.67 & 9.58 \\
13 & Nor-adrenaline 0.I\% injection & 16.67 & 8.33 \\
14 & $40 \%$ dextrose injection & 61.28 & 8.05 \\
\hline
\end{tabular}

Notes: I US $\$=39$ ETB, The average cost of the medicine per patient is obtained by dividing the sum of the medicine cost from total prescription by the number of prescriptions. 
high demand for medications that repeatedly make the pharmacies out of the products. For example, a participant shared:

I don't find drugs in the nearby pharmacies. If I get it one day, it might not be available the next day. There is a fluctuation in the availability of drugs even in the market. For example, Heparins' availability is inconsistent. If I find it one day, I may not find it the second day. Because of its demand, other patients will buy all of the available ones. (Male patient, 29yrs old)

Moreover, health care professionals were not aware of the availability status of medications in the hospital. They emphasized that they became aware of it through patients reporting back to them that they could not find the medicines. Through time they knew which medications are available or not, as patients came back with undispensed prescriptions repeatedly.

A pharmacist stated that the Drug Information Center tries to inform physicians about the availability status of medications through e-mail, but the system is not that functional. In addition, he also mentioned that the current stockout level of the hospital pharmacy in terms of items is considered severe.

We do not have third-generation cephalosporins, anti-biotics used for resistant conditions, blood-thinning medications, and injection medications for emergency purposes. As observed from this, the current stock out level is high.

\section{Management of the Burden of Stockout}

All the parties, including the patients and the prescriber, practised different approaches to managing the stockout and the medication's cost-related burdens. Patients said they borrowed money from their friends, close relatives, colleagues, and sometimes from other patients and/or caregivers to manage the costs of medications.

There was a medication we bought every day. It costs 1400 birr for a single daily dose. I've consulted the attending physician, but he said it is mandatory to take the medication. So, I borrowed money, since the hospital stay was for more than one month which I couldn't afford to buy all the doses. (Female caregiver,38 years old)

There were also instances they have to sell their assets to manage the costs of medications. The nurse also shared that borrowing medications among patients are a common trend in the ward, to the point it the nurses sometimes facilitate it.
Consultation of health care professionals about the stockout and the cost of the drugs was another mechanism to cope with the burden that the patient faces. Patients consult health care professionals in the ward always about the unavailability of medications. They often ask where they can find the medicines, what to do if they cannot afford to buy them, and ask for possible substitutions. The nurse mentioned that:

Almost all patients in the 36 beds complain of medication unavailability. It is rare to see someone not complaining. The most commonly available ones are Ceftriaxone and syringe if not finished for the day, so they will consult us on what to do.

The hospital pharmacy employs several strategies to tackle the consistent unavailability of medications, as shared by the pharmacists. These strategies include giving timely stock out information to the drug and therapeutics committee, requesting donors, forecasting, and communicating with other hospitals to borrow medications.

The other strategy employed by the participants to manage medication costs was to economize on different essential needs. Because health is their priority, often, patients forgo their basic needs. Skipping meals was common among the participants. A caregiver shared that she even cut back from her expenses aimed towards her children education.

I deducted from my children education tuition. There are even times where I have to take from my emergency savings. It has taken all I have. It is an extra expense. (Female caregiver, 40 years old)

Though not routine, the final strategy used by health care professionals working on the internal medicine ward is to contributed money to help patients who cannot afford to buy medications in circumstances where no options remain, and patients are obliged to buy medications outside. Besides this, professionals inform social care workers so that they help patients who cannot afford to pay for their medications.

\section{Impact of the Stock Out}

Delay in treatment and complications were mentioned as a significant outcome of stock out of drugs in the hospital pharmacy, and patients challenge to buy the drugs outside. All participants shared that medication unavailability has delayed their treatment. Extended hospital stays were primarily due to medication unavailability. Since patients 
went around the city looking for medications and waited some time by postponing doses till they get money to buy medicines, missing doses were common. One caregiver described a situation where her father experienced diseaserelated complication while she was searching for medication by saying:

When I went out to search for medications, I always left my father alone. There was a time where he got hypoglycemic and fainted. No one noticed him at the time. It was the nurse who discovered it when she came to give the afternoon medications. She gave him a soft drink, and he regained consciousness. I was not there when all this happened because I was in search of medications outside the hospital. (Female caregiver, 38 years old)

Delay of treatment was mostly accompanied by developing complications, particularly because of missed doses, but lengthened hospital stays. A resident mentioned that:

Most patients have developed septic shock and sepsis as a result of inappropriate treatment. I've witnessed many patients developing pulmonary embolism because of heparin unavailability. More complications will occur, and the patient will stay more than their expected length of stay. Complications might lead to death in some deplorable conditions.

The respondents mentioned suboptimal treatment as one of the outcomes of a shortage of medicine. As described by prescribing doctors, they always consider the economic status of patients when prescribing drugs that are not available within the hospital pharmacy. After consulting patients, a decision will be made. If possible, a less expensive effective substitute is used. However, there are cases where a replacement could not be an option, which necessitates patients to purchase the prescribed medication irrespective of their economic status. Not all patients are capable of buying these mandatory drugs; as a result, suboptimal treatment with the available medicines ensues. A physician exemplified this situation.

It has a major effect in the treatment process because patients may not get the standard treatment, even they might not get a replacement treatment if the drug is not available in the market even if they can afford it. As a health care professional, I am disappointed that we have to treat our patients with a substandard protocol because of unavailability of drugs; cheap to the expensive ones.
Time taking search of medications was another burden of unavailability of medicines in the hospital pharmacy, experienced by patients. The time required to purchase medicines outside the hospital pharmacy was from few hours up to days.

Searching for medications was the most tiring experience patients and their caregivers had during their hospitalization. Especially searching medicines at night were very exhausting, making patients feel bad for their caregivers. When patients do not have caregivers, they had to search for it by themselves, which was exhausting given their health condition. Participants shared they were wearied of searching to the point of giving up. This was supported by one patient as follows;

Climbing up these stairs by itself is tiring. I went back and forth ten times, and I got tired and stopped searching drugs one day. (Male caregiver, 40 years old)

Participants who were not aware of the prices of medications might not have the required amount of money to obtain their medications. They went several times back and forth between the hospital and the pharmacies.

I went to the pharmacies on foot because I didn't have money for transportation. When I reached there, the price of the medications was high. One day, I've gone back and forth three times on foot to collect money to buy the medications because what I've got was not enough. My wife also went back to 'Woliso' three times to bring money. She came back the day before yesterday and then went back today. (Male Caregiver, 40 years old)

In addition to impacts on physical health, patients and/or caregivers were under constant stress when managing their medication costs. The vicious cycle of stress from worries of unavailability of medications within the hospital pharmacy to additional expenses of medicines outside the hospital pharmacies occurred in patients and/or caregivers. It was evident from the patient's response that there was fear of worsening of symptoms due to missed doses, as some patients have experienced or seen others have lifethreatening symptoms. The following quote is illustrative of the stress experienced by caregivers.

I'm very stressed. I am becoming forgetful. Sometimes, I don't even pay attention during regular conversations. My wife went into depression and became an insomniac because we have spent a lot of money. I will get her treated when my child gets discharged. (Male caregiver, 49 yrs old) 
The resident shared her peculiar experience with a patient:

I had one patient that has spent 130,000 birrs for antibiotics, particularly meropenem, for only 15 days. The patient is from a rural area. The prognosis was poor, and even after spending all that, the patient died.

Having mentioned the entire burden and the impact of the stockout and medication cost outside of the hospital pharmacy, all the parties, forwarded their recommendations. Patients have raised their recommendations by stating that the hospital should avail medications as it is the biggest health institution in the country. They strongly believe that the hospital is capable of purchasing the necessary medicines. In cases where the hospital has constraints in availing medications, giving financial support for patients who cannot afford to buy medications was also another point raised from patients and/or caregivers' side.

From a professional side, subsidizing the hospital to bring an efficient system that avails all the necessary medications was the common recommendation shared. Both the physicians and pharmacists stressed that the national essential drug list that does not contain the unique medications TikurAnbessa Specialized Hospital uses as a tertiary hospital is outdated and needs to be updated.

The most significant hurdle that hindered the hospital pharmacy from availing medications as shared by the pharmacist was the absence of a separate entity led by professional pharmacists to purchase medicines, resulting in an inefficient procurement system that uses the hospital budget. There is an initiative to open a community pharmacy that utilizes a revolving drug fund system, which makes the hospital pharmacy use its budget to procure medications. This is believed to decrease the existing burden of unavailability of medicines at the hospital.

\section{Discussion}

Unavailability of drugs have adverse economic, clinical and humanistic outcomes to patients. ${ }^{5}$ Patient are resorted to buy medications outside the hospital pharmacy that exposes patients to several burdens associated with cost and health condition.

As shown in this study only $38.23 \%$ of medications prescribed were available within the hospital pharmacy during the study period, which is higher than the finding in the pharmaceutical level assessment of Uganda which is $24.7 \%$ mean availability of medications in public sectors. ${ }^{6}$

A qualitative study that assessed parents' and guardians' perceptions on availability and pricing of medicines and healthcare for children in eThekwini, South Africa, reported that the majority of users complained that availability of drugs and vaccines was problematic in the public sectors and were sometimes forced to use private-sector pharmacies due to shortages or unavailability of drugs at the public sector clinics. ${ }^{7}$

Prescribers in TASH internal medicine ward get stockout information from the patient. They said those patients who were not able to get the prescribed drugs returned to them, in this process, they get the stock out information. They mentioned that the unavailability of TASH is a severe problem and ultimately affects patient treatment outcomes. This finding was similar to another study done in Ethiopia, where it stated that the physicians working in a public hospital (96\%) strongly or partly agreed there is a lack of enough resources to provide standard medical care in public hospitals in Ethiopia. ${ }^{8}$

The total costs of medications bought inside and outside the hospital pharmacy were 78.07 and $1530.84 \$$, respectively.

Hence, the patients' cost for medication outside was nearly 20 times higher than TASH pharmacy. Similarly, a study on the availability and affordability of essential medicines for children in the Western part of Ethiopia founded that patient prices were $36 \%$ times higher in the private sector than in the public sector, the unaffordability of lowest-priced medicines varying from 1.5 to 8.7 days' wages, and 1.8 to 30.7 days' wages in the public and private sector respectively. ${ }^{9}$ Therefore, the lack of medication in the hospital pharmacy put the patients and the caregivers for unnecessary and unreasonable burden.

Regarding affordability irrespective of the socioeconomic level, all participants in the qualitative study in South Africa mentioned that medicines were expensive, although considered affordable by the middle class and affluent groups. The study observed the vast difference in total costs of medications inside and outside pharmacies. ${ }^{7}$

The total medication cost differences within and outside the hospital pharmacy were enormous. The total cost of medications outside the hospital pharmacy was nearly 20 times higher than the cost of medication inside the hospital pharmacy, whereas the average cost is 12 folds greater. This study shows how patients are exposed to huge expenditure as a result of medication unavailability in TASH. Moreover, it leads the patients to unnecessary stress and unsatisfied diseases progression. In this study, most the patients had less than 1000ETB monthly income, and almost, $81 \%$ of the respondents had less than $102.56 \$$ 
monthly income. But on average, they need $54.67 \$$ to purchase medication from outside private pharmacies and $4.34 \$$ to purchase drugs from TASH for an average of 8 days of dose. So, the unavailability of drugs in the hospital puts the patients on a heavy burden beyond their monthly earnings and forces them to borrow money and sell their property.

Unfractionated Heparin was one of the costliest among the medication bought from outside pharmacies. The cost of Heparin ranged from $0.076 \$-0.38 \$$ when it was available within the hospital pharmacy, making the current average cost per patient to be 39 to 198 folds higher. Since most of the admitted patients needed Heparin as prophylactic or treatment medication, this put the patients for unwanted and unreasonable spending of their money. This particular finding shows how the unavailability of medications affects the price of the drugs dramatically.

In addition to costs of medications, several indirect costs were incurred by patients. Transportation costs and lengthened hospital stay which in turn decreases productivity of patients and/or caregivers. Direct medical costs besides medication costs were also considerable as patients shared laboratory tests and costs of medical supplies and equipment were intensive.

Physicians always consider the economic status of patients when prescribing medications that are not available within the hospital pharmacy, where suboptimal treatment in sues in cases where patients cannot afford to buy medicines. These strategies employed by physicians were in line with the findings of a study on how Ethiopian physicians try to minimize out-of-pocket health expenditures, as $88 \%$ of physicians reported costs for the patient to be important when deciding to use or not use an intervention. In addition, $73 \%$ of physicians felt obliged to protect against out-of-pocket expenses by recommending cheaper, but second-best treatment. ${ }^{8}$ Another study done on pharmacy director members shows $48.5 \%$ of suboptimal treatment. ${ }^{10}$

Patients have several strategies to cope with the medication cost burden, such as borrowing money, selling assets, cutting back on general needs, and consulting health care professionals. These findings were in line with a qualitative study assessing medication cost burden in patients with disabilities in Canada. Moreover, several impacts such as patients and/or caregiver exhaustion, exacerbations of symptoms, lengthened hospital stay, and psychological stress was also supported by study. ${ }^{11}$
Likewise, qualitative findings on impacts of medication unavailability on patients were supported by quantitative results of a survey on effects of drug shortages on patient care stating that the most common patients' outcomes reported by respondents were alternative medication used $[\mathrm{n}=146,85.3 \%]$, and delay of therapy $[\mathrm{n}=121,70.8 \%]^{10}$

\section{Limitation of the Study}

Short data collection period and the small sample size is the major limitation of this study. The study was also conducted in only one ward of internal medicine ward, which might affect the generalizability. In this study, the cost was measured only for medication, and other indirect costs were not included in this study.

\section{Conclusion}

The unavailability of pharmaceuticals at the TASH internal medicine ward has caused patients to face many difficulties. From which economic, psychological stress and compromised treatment outcomes are the major ones. Patients spend extra money when the medications are unavailable in the hospital, and it also affects the prescriber to substitute the medicines.

In conclusion, delayed treatment outcomes, unwanted high spending to purchase pharmaceuticals from outside pharmacies are the major complaints associated with the unavailability of medication in the hospital pharmacy.

\section{Ethical Consideration}

Ethical clearance was obtained from the ethical review board of the School of Pharmacy, Addis Ababa University (ERB/SOP/212/11/2010). The study setting is a teaching hospital where scholars, clinicians, and students are allowed/accepted to request patients' information for academic and research purposes. The verbal informed consent was approved by the ethical review board, and that the informed consent included the publication of anonymized responses.

Since the study did not take samples from human participants, only verbal consent was taken from each patient, caregiver, and healthcare professionals before the commencement of data collection.

All study participants including caregivers, parent or legal guardian of participants under 18 years of age were informed about the purpose of the study and their participation was completely voluntary while confidentiality and anonymity were maintained. The study was conducted according to the Declaration of Helsinki. 
Due to the current global pandemic and the study being conducted in the COVID-19 era, all possible national infection prevention and control measures have been taken to avoid possible disease transmission.

\section{Acknowledgment}

I would also like to express my deepest gratitude and appreciation to the participants of the study for their cooperativeness, kindness, support, and family-like relation amidst their illness and stressful condition that has encouraged me a lot in the research process.

\section{Disclosure}

The authors report no conflicts of interest for this work.

\section{References}

1. Federal Democratic Repulic of Ethiopia Ministry of Health. Ethiopian hospital services transformation guidelines chapter 10: pharmacy service; 2017. Available from: https://www.ghsupplychain.org/sites/ default/files/2018-11/Pharmacy\%20Services\%20-\%20Hospitals\% 20Management\%20Initiative.pdf. Accessed August 9, 2021.

2. Wagner AK, Graves AJ, Reiss SK, Lecates R, Zhang F, Ross-degnan D. Access to care and medicines, burden of health care expenditures, and risk protection: results from the World Health Survey. Health Policy (New York). 2011;100(2-3):151-158. doi:10.1016/j. healthpol.2010.08.004
3. Geremew M, Gedefaw M, Tsegay G, Kassa GM, Info A. Magnitude of out of pocket health expenditures and associated factors among civil servants. Int J Public Health Sci. 2015;4(4):332-337.

4. Ongarora D, Karumbi J, Minnaard W, Abuga K, Okungu V. Medicine prices, availability, and affordability in private health facilities in low-income settlements in Nairobi County, Kenya. Pharmacy. 2019;7:40. doi:10.3390/pharmacy7020040

5. Phuong JM, Penm J, Chaar B, Oldfield LD, Moles R. The impacts of medication shortages on patient outcomes: a scoping review. PLoS One. 2019;14(5): 0215837.

6. The Repulic of Uganda Ministry of Health. Pharmaceutical situation assessment - level II; August, 2008. Available from: http://digicollec tion.org/hss/en/m/abstract/Js16377e/. Accessed August 9, 2021.

7. Perumal-pillay VA, Suleman F. Parents ' and guardians ' perceptions on availability and pricing of medicines and healthcare for children in eThekwini, South Africa - a qualitative study. BMC Health Serv Res. 2017;17:1-11. doi:10.1186/s12913-017-2385-y

8. Id IM, Defaye FB, Wakim P, et al. Financial risk protection at the bedside: how Ethiopian physicians try to minimize out-of- pocket health expenditures. PLoS One. 2019;14(2):e0212129.

9. Sado E, Sufa A. Availability and affordability of essential medicines for children in the Western part of Ethiopia: implication for access. BMC Pediatr. 2016;16:1-8. doi:10.1186/s12887-016-0572-3

10. Mclaughlin M. Effects on patient care caused by drug shortages: a survey. J Manag Care Pharm. 2013;19(9):783-788.

11. Gupta S, McColl MA, Guilcher SJT, Smith K. Managing medication cost burden: a qualitative study exploring experiences of people with disabilities in Canada. Int J Environ Res Public Health. 2019;16 (17):3066. doi:10.3390/ijerph16173066
Patient Preference and Adherence

\section{Publish your work in this journal}

Patient Preference and Adherence is an international, peer-reviewed, open access journal that focusing on the growing importance of patient preference and adherence throughout the therapeutic continuum. Patient satisfaction, acceptability, quality of life, compliance, persistence and their role in developing new therapeutic modalities and compounds to optimize clinical outcomes for existing disease states are major areas of interest for the journal. This journal has been accepted for indexing on PubMed Central. The manuscript management system is completely online and includes a very quick and fair peer-review system, which is all easy to use. Visit http:// www.dovepress.com/testimonials.php to read real quotes from published authors. 